\title{
Discrepancia taxonómica y riqueza de corales zooxantelados del Pacífico oriental durante el Cenozoico
}

\section{Taxonomic distinctness and richness of eastern Pacific zooxanthellate corals during the Cenozoic}

\author{
Héctor Reyes-Bonilla ${ }^{1}$, Ramón Andrés López-Pérez ${ }^{2 \bowtie}$ y Alonso Mohedano ${ }^{1}$

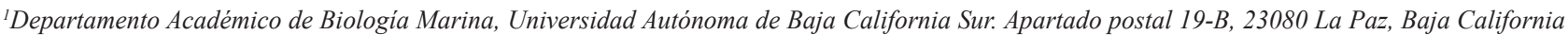 \\ Sur, México. \\ ${ }^{2} I n s t i t u t o$ de Recursos, Universidad del Mar, Campus Puerto Ángel. Apartado postal 47, 70902 Puerto Ángel, Oaxaca, México. \\ \alopez@angel.umar.mx
}

\begin{abstract}
Resumen. La fauna de corales zooxantelados del Pacífico oriental ha sufrido cambios ocasionados por extinciones, especiación e inmigración desde varias provincias zoogeográficas. Aquí se analizan los cambios en la riqueza y en la discrepancia taxonómica y su varianza $(\Delta+\mathrm{y} \Lambda+$, indicadores de la biodiversidad que cuantifican la longitud del árbol taxonómico), para los corales del oeste de las Américas durante el Cenozoico, a partir de un análisis de la presencia temporal de 148 especies, en intervalos de un millón de años. La discrepancia taxonómica fue muy estable durante el Cenozoico (alrededor de 44-50 unidades), mientras que la riqueza fluctuó de 42 especies en el Eoceno, hasta 3 en el Mioceno; esto indica que $\Delta+$ no se afectó con las oscilaciones en el número de especies. Por otro lado, la riqueza y la varianza de la discrepancia taxonómica se comportan de manera inversa, dado que en intervalos temporales con alto número de especies, éstas pertenecieron a grupos taxonómicamente afines. Finalmente, la comparación de $\Delta+\mathrm{y}$ $\Lambda+$ indicó que los índices se asocian negativamente. Así, es posible que la redundancia ecológica sea alta y menor la tolerancia a cambios ambientales cuando es mayor la discrepancia taxonómica, dado que las especies están arregladas en un reducido número de géneros o familias.
\end{abstract}

Palabras clave: Scleractinia, extinción, arrecifes de coral, diversidad biológica, fósil, Cenozoico.

\begin{abstract}
The zooxanthellate coral fauna of the eastern Pacific has changed due to mass extinctions, speciation and immigration from several zoogeographic provinces. In this study we analyze changes in richness and taxonomic distinctness and variance $(\Delta+\mathrm{y} \Lambda+$, biodiversity indicators that quantify the length of the taxonomic tree of the species) for stony corals of western America during the Cenozoic, on the basis of an analysis of the temporal occurrence of 148 species, arranged in one-million year intervals. Taxonomic distinctness was very stable during the Cenozoic (around 44-50 units), while species richness fluctuated from 42 species in the Eocene to 3 in the Miocene; this indicates that $\Delta+$ was not affected by oscillations in species richness. On the other hand, richness and taxonomic distinctness variance behave inversely, due to the fact that in times with high species number, they belonged to similar higher taxonomic groups. Finally, a comparison of $\Delta+$ and $\Lambda+$ showed that they are negatively associated. Thus, it is feasible that there might be higher ecological redundancy and less tolerance to environmental change in time periods with high taxonomic distinctness, as the species are arranged in a reduced number of genera and families.
\end{abstract}

Key words: Scleractinia, extinction, coral reefs, biological diversity, fossil, Cenozoic.

\section{Introducción}

La región del Pacífico oriental tropical es una de las más pobres del mundo en lo referente a la diversidad de corales zooxantelados, debido a que las condiciones ambientales de la costa oeste del continente americano no son idóneas para el desarrollo de escleractinios (Glynn y Ault, 2000); el litoral presenta amplias zonas arenosas, alta productividad fitoplanctónica, aguas frías, gran acidez, y una angosta franja de plataforma continental somera, lo

Recibido: 20 mayo 2011; aceptado: 09 marzo 2012 cual dificulta el crecimiento de los arrecifes. No obstante, la fauna coralina ha recibido gran atención de los zoogeógrafos por estar constituida fundamentalmente de especies no nativas, colonizadoras desde el Pacífico central y occidental (Glynn, 2001; López-Pérez, 2005), lo que posibilita encontrar una mezcla de corales endémicos descendientes de faunas del océano Atlántico junto con especies que han evolucionado en el Pacífico de manera independiente desde el cierre del mar de Tethys, hace 37-24 millones de años (Veron, 1995).

La historia de los corales del Pacífico oriental en el Cenozoico está relativamente bien caracterizada (Colgan, 
1990; López-Pérez, 2005, 2008; López-Pérez y Budd 2009) y ha pasado por 3 fases desde la perspectiva de afinidad biogeográfica de sus faunas. Entre el Paleoceno y el Oligoceno presentaba alta riqueza, los arrecifes ocupaban zonas de alta latitud (hasta el sur de Canadá) y estaba formada en gran medida por especies e incluso géneros endémicos (Durham, 1947). Para el Mioceno y el Plioceno temprano, las faunas cambian y pasan a parecerse a las que ocupan la región del Atlántico occidental, ya que en ese entonces la comunicación interoceánica estaba totalmente abierta; no obstante, la riqueza siguió siendo menor en la costa del Pacífico por la presencia de zonas de surgencia a lo largo de la costa (Colgan, 1990). Finalmente, hace aproximadamente 3.2 millones de años se cierra el Portal de Bolívar y las faunas del Pacífico americano quedan aisladas del Atlántico (Veron, 1995). Ese evento produjo un aumento en la tasa de extinción local, casos de especiación (por ejemplo, el surgimiento de Porites sverdrupi Durham, 1947; López-Pérez y Budd, 2009) y un fuerte enriquecimiento faunístico por colonización a larga distancia, originado por el cambio en dirección y velocidad de las corrientes en el océano Pacífico y el consecuente incremento en el transporte larval (Grigg y Hey, 1992). Así, para el Pleistoceno medio ( $c a .0 .3 \mathrm{ma}$ ) existían poblaciones de especies de afinidad indo pacífica en la costa oeste de México (López-Pérez, 2008; López-Pérez y Budd, 2009) y en la actualidad, el proceso de colonización ha continuado, siendo especialmente intenso el transporte de larvas en los años donde se presenta el fenómeno de Oscilación Sureña El Niño (Glynn, 2001).

Los patrones macroevolutivos de las faunas coralinas han sido estudiados extensivamente para la región del mar Caribe (Budd, 2000; Johnson et al., 2008; Budd y Pandolfi, 2010) y a una escala mundial para la interfase CretácicoTerciario (Kiessling y Baron-Szabo, 2004; Baron-Szabo, 2008; Pandolfi, 2011), pero aún hay pocos datos sobre el tema para el Pacífico oriental. Al respecto, López-Pérez y Reyes-Bonilla (2000), López-Pérez (2005) y López-Pérez y Budd (2009) indicaron que la tasa de extinción ha sido superior a la de originación en gran parte del Cenozoico, razón por la cual la riqueza de especies ha ido en decremento durante los últimos millones de años. Con el fin de aportar nuevos datos respecto a la dinámica evolutiva de los corales del Pacífico oriental, en el presente trabajo se revisaron los cambios y patrones temporales de la riqueza de especies durante el Cenozoico y se analizó la respuesta de parte de la discrepancia taxonómica de las comunidades coralinas $(\Delta+$; un componente de la biodiversidad ligado a la distancia taxonómica promedio entre especies) y de su variabilidad $(\Lambda+)$. La hipótesis de trabajo fue que las notables fluctuaciones registradas en la diversidad de escleractinios durante el Cenozoico se han visto también reflejadas en indicadores que cuantifican distintas expresiones de la biodiversidad.

\section{Materiales y métodos}

Para el presente estudio se construyó una base de datos de 148 especies de corales zooxantelados fósiles y recientes, distribuidos en el Pacífico oriental tropical (entre Alaska y Chile) durante los últimos 65 millones de años. La información se generó a partir de la revisión de ejemplares depositados en las siguientes instituciones: Paleontology Repository, Department of Geoscience, University of Iowa; National Museum of Natural History, Smithsonian Institution; Natural History Museum of Los Angeles County; Yale Peabody Museum; Santa Barbara Museum of Natural History; University of California Museum of Paleontology; Museo de Historia Natural de la Universidad Autónoma de Baja California Sur. También se incorporaron registros de ejemplares recolectados en el golfo de California por el segundo autor (Mioceno tardíoReciente) y determinados mediante taxonomía tradicional y geométrica (López-Pérez, 2008, 2012), además de datos obtenidos de literatura primaria (López-Pérez, 2005). Los nombres válidos y las sinonimias se establecieron siguiendo los criterios de Vaughan (1917), Foster (1986, 1987), Budd (1991), Budd y Johnson (1999), ReyesBonilla (2002), López-Pérez (2012), Reyes-Bonilla et al. (2005), López-Pérez y Budd (2009). La edad relativa y/o absoluta de los afloramientos fósiles se determinó mediante revisiones bibliográficas, relaciones estratigráficas y razones isotópicas de uranio y torio (López-Pérez 2008, López-Pérez y Budd 2009). La duración temporal de cada especie se determinó en función de la edad mínima y máxima de cada afloramiento; por ejemplo, la edad para la Formación Carmen y Marquer, donde se colectaron Porites panamensis Verrill, 1866, Pocillopora capitata Verrill, 1864, Favia maitreyiae López-Pérez, 2012, Porites carrizensis Vaughan, 1917 y Dichocoenia merriami (Vaughan, 1900) es de 3.1-1.8 m.a. (véase López-Pérez, 2008) y por ello, la duración de cada especie se considera de 3 periodos de 1 millón de años (Apéndice 1). Si bien esta aproximación tiende a sobreestimar la duración temporal de los taxa, es preferible a otras de su tipo (i.e., asignar la duración de los taxa a la parte media del intervalo de edad del afloramiento de su presencia), sobre todo cuando no se puede afirmar que la verdadera edad del afloramiento se encuentra restringida a ese intervalo temporal (Johnson, 2001). Finalmente, cabe mencionar que aún cuando se ha avanzado en el conocimiento sobre la taxonomía y la determinación de la edad de los taxa coralinos que han habitado en el Pacífico oriental tropical durante el Cenozoico y particularmente de aquellos recolectados en 
el golfo de California (Johnson et al., 2007; López-Pérez, 2008; López-Pérez y Budd, 2009) y Chiapas (Budd, 2000), la situación respecto a los ejemplares recolectados en los estados de Washington y California (Estados Unidos de América) con una edad entre Paleoceno y Oligoceno, merecen un estudio mucho más detallado.

A partir de la base de datos se calculó la discrepancia taxonómica para cada intervalo de tiempo, usando el índice $\Delta+$ de Warwick y Clarke (1995). Este indicador evalúa la distancia taxonómica promedio (definida a través de un árbol de clasificación Linneana) entre pares de especies elegidas al azar en una asociación y es análogo a los de diversidad filogenética (Schweiger et al., 2008), por lo que ha sido considerado como un estimador más preciso de la biodiversidad sensu lato que los medidores ecológicos tradicionales (Magurran, 2004). El índice posee, entre otras, las virtudes de no sufrir sesgos por la riqueza específica o por el esfuerzo de muestreo (Warwick y Clarke, 1998); ésto representa una gran ventaja para el trabajo paleontológico, donde es difícil controlar el tamaño de muestra. Además, a manera de complemento se calculó el índice de variación de la discrepancia taxonómica $(\Lambda+)$, el cual es análogo a una "varianza taxonómica" y define la equidad en la estructura del árbol taxonómico, es decir, la homogeneidad en la representación relativa de grupos supraespecíficos en la fauna (Clarke y Warwick, 2001). La forma de respuesta del indicador es tal que, en el caso de igual riqueza, alcanza cifras más bajas cuando la mayoría de especies en la colección de datos pertenece al mismo grupo taxonómico mayor (género o familia en este caso) y toma valores elevados mientras mejor distribuida esté la riqueza entre grupos (Warwick y Clarke, 2001). Una vez con los valores de $\Delta+$, $\Lambda+$ y la riqueza específica de corales se evaluó la relación entre estos 3 indicadores a lo largo del tiempo (millones de años), mediante el coeficiente de correlación por rangos de Spearman (Zar, 2009). Este procedimiento se llevó a cabo dada la falta de normalidad de los datos de riqueza y discrepancia taxonómica y sólo se aplicó a los intervalos de tiempo donde se registró al menos una especie ( $56 \mathrm{de}$ 65 totales), ya que la nula riqueza conocida actualmente para esos lapsos pudiera ser en realidad un artefacto que no indica la extinción total de la fauna coralina arrecifal en la región, sino la carencia de afloramientos representativos durante el intervalo de tiempo.

\section{Resultados}

La figura 1 muestra que la riqueza de especies de coral ha tenido fuertes fluctuaciones en el Cenozoico, con los mayores valores en intervalos de tiempo distribuidos en el Reciente (42 especies), Oligoceno (24-27 m.a.; 37 especies) y Eoceno (41-45 m.a.; 32 especies); en contraste, los más bajos se presentaron al principio de esta era, entre los 64-55 m.a. (Paleoceno-Eoceno; 3 especies), así como en la transición Eoceno-Oligoceno (32 m.a.) y el Mioceno (14-11 m.a.), donde sólo se tiene registro de una especie. Esos últimos eventos resultan de una serie de extinciones regionales que ocurrieron en las mismas épocas (Eoceno $\sim 40$ m.a.; Oligoceno-Mioceno, 23-24 m.a.; finales del Mioceno, 7-6 m.a). Finalmente, cabe señalar que en 9 intervalos (7-10, 22 y 36-39 m.a.), no existen formaciones donde se hallan registrado especies de corales zooxantelados en la costa oeste de las Américas, lo que no significa que la diversidad haya sido de cero; simplemente aún se carece de datos al respecto.

En claro contraste con los hallazgos descritos, la discrepancia taxonómica $(\Delta+)$ ha sido muy estable y permanece entre las 38 y 49 unidades, aunque hace 23 m.a. (Oligoceno-Mioceno) se presentó un valor mínimo de 33.3 unidades como resultado de una extinción coralina que sólo dejó 4 especies de 2 géneros (Siderastrea y Eusmilia; véase López-Pérez, 2005; Fig. 1). Este es el único caso que se vio claramente reflejado tanto en la riqueza como en $\Delta+$. Finalmente, la tendencia de la variación de la discrepancia taxonómica no coincidió con la de los índices de biodiversidad anteriores (Fig. 2), ya que las cifras más altas $(\Lambda+>140)$ se presentaron en el Eoceno (55-64 m.a.), Oligoceno (a los 23 m.a.) y en el Reciente, mientras que el resto del Cenozoico el indicador varió entre 25 y 93 unidades. Además, a los 23 m.a. y entre 11-14 m.a. (todo durante el Mioceno), el índice tomó valores de cero porque en los afloramientos fósiles sólo se ha registrado una especie y por ende, no existe varianza en los datos (Clarke y Warwick, 2001).

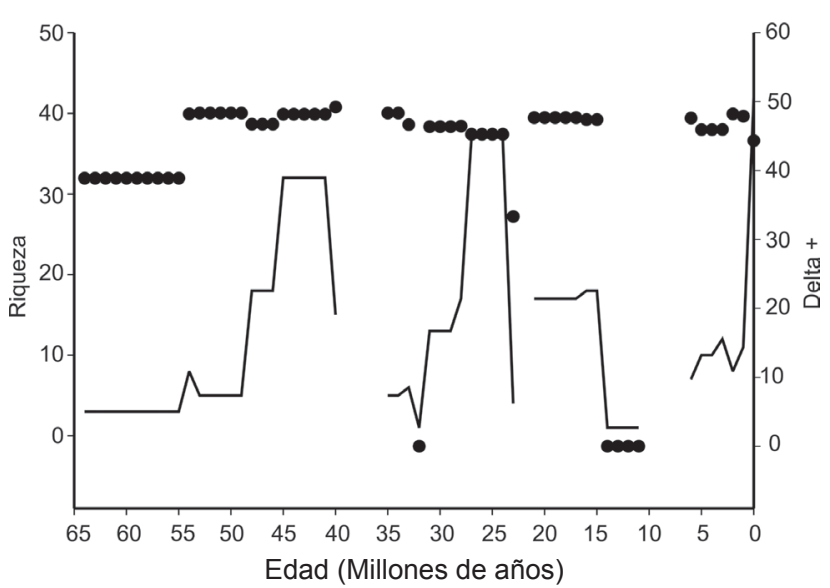

Figura 1. Variación de la riqueza de especies y discrepancia taxonómica $(\Delta+)$ de los corales zooxantelados del Pacífico americano durante el Cenozoico. Línea= riqueza de especies, puntos $=$ discrepancia taxonómica $(\Delta+)$. Obsérvese la diferencia de escala para cada índice. 


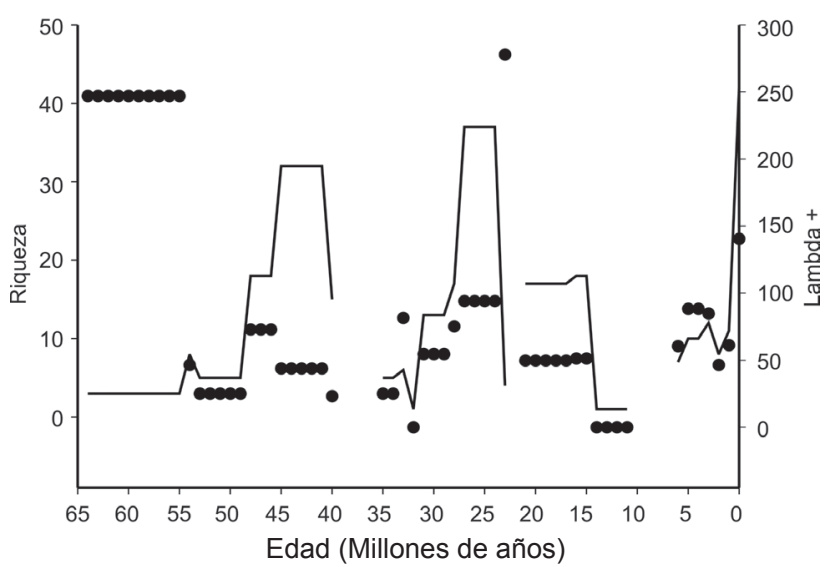

Figura 2. Variación de la riqueza de especies y la variación de la discrepancia taxonómica $(\Lambda+)$ de los corales zooxantelados del Pacífico americano durante el Cenozoico. Línea= riqueza de especies, puntos $=$ variación en la discrepancia taxonómica $\left(\Lambda^{+}\right)$. Obsérvese la diferencia de escala para cada índice.

Al revisar las relaciones entre índices, la figura 1 muestra que la riqueza de especies ha disminuido sensiblemente a lo largo del Cenozoico (Spearman $\mathrm{r}=-0.561$, $\mathrm{n}=51, p<0.001$ ), además de que al principio y al final de este periodo se comportó de manera similar a la discrepancia taxonómica (es decir, intervalos de tiempo con bajo número de especies presentaron la menor longitud promedio del árbol taxonómico y a la inversa). Sin embargo, el análisis de correlación entre la riqueza y la discrepancia taxonómica señaló que dicha relación no es estadísticamente consistente (Spearman $\mathrm{r}=0.255, \mathrm{n}=51, p=0.070$ ), lo que evidencia la independencia en la respuesta de los indicadores de biodiversidad citados. En contraste, $\Lambda^{+}$y la riqueza están ligados de forma negativa (Spearman $\mathrm{r}=$ $-0.282, \mathrm{n}=51, p=0.044$. Fig. 2), lo que apunta que intervalos de tiempo con mayor número de especies presentaron un arreglo menos homogéneo en su distribución dentro de taxa mayores. Así, puede decirse que en tiempos de alta diversidad de corales zooxantelados en el Pacífico oriental, las especies tienden a pertenecer a los mismos grupos taxonómicos superiores.

Por otra parte, la comparación de los valores de $\Delta+\mathrm{y}$ el tiempo geológico (Spearman $\mathrm{r}=-0.173, \mathrm{n}=51, p=0.225$. Fig. 1) pone de manifiesto una conexión negativa aunque no significativa entre ambos. En el caso de $\Lambda+$, este indicador era más alto a principios del Cenozoico y su valor también ha ido disminuyendo, pero de nuevo la relación no es estadísticamente confiable (Spearman $\mathrm{r}=0.159, \mathrm{n}=51$, $p=0.263$. Fig. 2). Finalmente, ambos índices están fuertemente asociados entre sí y se comportan de forma inversa (Spearman $\mathrm{r}=-0.981, \mathrm{n}=51, p<0.0001$ ), indicando que en aquellos periodos cuando la discrepancia taxonómica $(\Delta+)$ es mayor, las especies de los ensamblajes tienden a formar parte de los mismos grupos mayores (géneros o familias).

Recapitulando, los resultados del estudio indican que: a) la riqueza de especies de corales zooxantelados en el Pacífico oriental ha fluctuado notablemente durante el Cenozoico; b) la discrepancia taxonómica $(\Delta+)$ ha sido relativamente invariable en el tiempo y sus valores están desacoplados de las modificaciones en la riqueza específica y c) la variación de la discrepancia taxonómica $(\Lambda+)$ disminuye tanto con la riqueza como con $\Delta^{+}$, lo que marca una tendencia hacia la mayor presencia de especies emparentadas (pertenecientes al mismo grupo taxonómico mayor) cuando la diversidad ha sido mayor.

\section{Discusión}

El presente estudio muestra que la riqueza específica de corales zooxantelados del Pacífico oriental ha sufrido variaciones importantes a lo largo del tiempo geológico y tiene una tendencia neta a disminuir hacia el presente (Fig. 1), lo que ya antes López-Pérez (2005) había registrado. También se había observado a escala de géneros desde Durham (1947) y con datos más recientes por Colgan (1990) y por Grigg y Hey (1992). Dichos autores han indicado que esos cambios resultaron de una serie de eventos de extinción en el Eoceno, Oligoceno-Mioceno y Mioceno-Plioceno; además, por el alto endemismo que ocurrió en el Eoceno y un pulso de colonización desde el Pacífico central en el Reciente. A pesar de que las fluctuaciones están claramente definidas en términos de riqueza, no se han visto reflejadas en el índice de discrepancia taxonómica (el cual se ha mantenido relativamente equilibrado en el tiempo; Fig. 1), y ambos muestran desfases en su comportamiento, en especial durante los últimos 30 millones de años. Para explicar estos patrones se requiere revisar el significado ecológico del indicador de diversidad taxonómica empleado.

Clarke y Warwick (1998) observaron que para que el nivel de $\Delta+$ sea persistente, no debe haber modificaciones importantes en la proporción del número de taxa relativo a su nivel inmediato superior (especies en cada género; géneros por familia, etc.). Al revisar la situación de los corales arrecifales del Pacífico en el Cenozoico (Fig. 3) se advierte que la relación especies/géneros y géneros/familias fluctúa de 1.0 a 2.0, con apenas algunos valores atípicos superiores a 2.0 entre los 23 y 27 m.a. y en el Reciente (Fig. 3). En función de ese comportamiento puede sugerirse que la constancia en la relación de la riqueza entre distintos niveles de la jerarquía taxonómica ha dado lugar a que la longitud media del árbol taxonómico de los corales no varíe mucho, $\mathrm{y}$ consecuentemente a que $\Delta+$ haya sido relativamente estable 
durante el Cenozoico e independiente del número de especies presentes y de la edad geológica.

Bajo otra perspectiva, se ha visto que la discrepancia taxonómica responde de manera muy parecida a la diversidad filogenética (Schweiger et al., 2008) y ambas son marcadores eficientes de la variedad de funciones ecológicas de una comunidad, en la lógica de que mientras más separadas filética y taxonómicamente estén las especies, su morfología, sus características biológicas y sus nichos ecológicos diferirán en mayor medida (Rogers et al., 1999). Esta propiedad de $\Delta+$ tiene gran relevancia para el análisis macroevolutivo de la fauna de escleractinios, pues si se considera que los valores del indicador variaron muy poco en el Pacífico oriental, ello sugiere que este grupo ha mantenido un ámbito relativamente constante de desempeño funcional durante el Cenozoico, aún en momentos particularmente álgidos y de crisis biológica (Fig. 1). Los resultados del índice contrastan con lo observado para las asociaciones de los mares Caribe y Atlántico occidental, donde las extinciones de corales arrecifales han sido selectivas durante los últimos 65 m.a. y han afectado la funcionalidad de esos ecosistemas (Budd, 2000). La relación entre $\Lambda+\mathrm{y}$ la riqueza de especies demostró que ambos varían significativamente y de forma inversa (Fig. 2), lo que implica que en épocas cuando se registra alto número de corales zooxantelados en el Pacífico oriental, la región ha estado dominada por elementos taxonómicamente más emparentados, es decir, pertenecientes a los mismos géneros o familias (Warwick y Clarke, 2001). Este comportamiento puede observarse incluso en las comunidades recientes del oeste de las Américas (Reyes-Bonilla, 2002), ya que aunque se tiene una de las más altas riquezas registradas históricamente, el elenco está dominado por los géneros Pocillopora

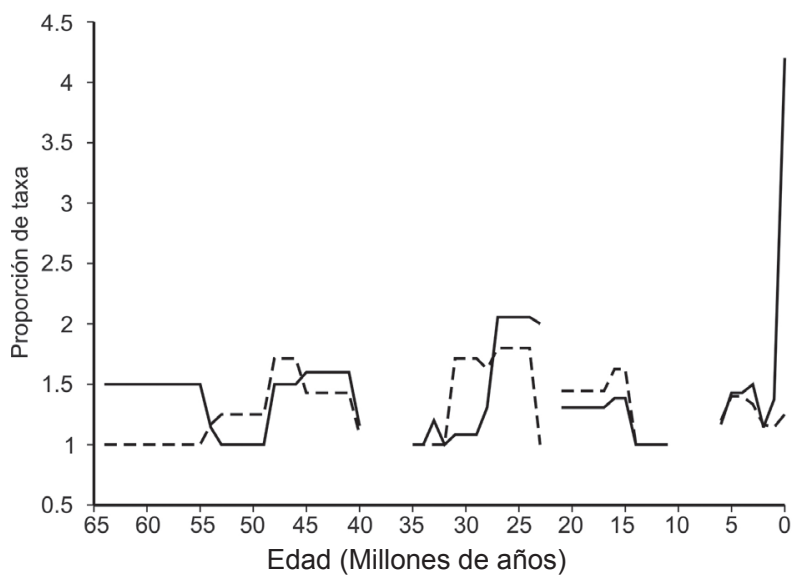

Figura 3. Proporción de especies por género y géneros por familia para corales zooxantelados en el Pacífico oriental durante el Cenozoico. Línea= especies/género, línea punteada= géneros/ familia.
(Familia Pocilloporidae; 9 especies), Porites (Poritidae; 9 especies) y Pavona (Agariciidae; 7 especies). De manera análoga, durante el otro pico de diversidad, a mediados del Eoceno (41 a 45 m.a.), López-Pérez (2005) indicó que el $35 \%$ de la fauna regional la constituyeron especies pertenecientes a los géneros Astreopora, (familia Acroporidae) Madracis (Pocilloporidae) y Haimesiastrea (Columastreidae). Que durante intervalos de tiempo particularmente diversos para la fauna de corales, los taxa pertenezcan a un pequeño número de grupos supraespecíficos, puede deberse a las condiciones ambientales prevalecientes durante los últimos 65 m.a. en la costa oeste del continente americano que no han sido las óptimas para el grupo (Veron, 1995; Glynn y Ault, 2000). Así, el entorno debe ejercer mecanismos activos de selección de especies que han favorecido sólo a aquellas que han contado con las características necesarias para colonizar, asentarse y construir las relativamente modestas estructuras arrecifales que se han desarrollado a lo largo del Cenozoico. Finalmente, aun cuando $\Delta^{+}$y $\Lambda^{+}$ son calculados a partir del mismo grupo de datos, no existe ninguna correlación intrínseca entre ambos debido a que los índices rescatan información independiente (Warwick y Clarke, 1998), aunque no es raro que los dos presenten tendencias estadísticamente significativas (Clarke y Warwick, 2001). La fuerte relación negativa que se observó durante el presente estudio es producto del citado incremento relativo en el número de taxa supraespecíficos (valores altos de $\Delta+$ ) acoplado a un incremento de elementos emparentados taxonómicamente (valores reducidos de $\Lambda+$ ).

Las investigaciones realizadas en comunidades marinas y dulceacuícolas actuales han mostrado que los valores bajos de discrepancia taxonómica y de su variación $(\Delta+\mathrm{y}$ $\left.\Lambda^{+}\right)$son una respuesta común a distintos grados de disturbio (Graham et al., 2006; Campbell y Novelo-Gutiérrez, 2007) y en muchas ocasiones, implican reducción en el número de grupos funcionales de una comunidad (Warwick y Clarke, 2001; Euler y Svensson, 2001). En este sentido, la relación inversa entre $\Lambda+$ y la riqueza pueden tomarse como señal de que las comunidades ricas en escleractinios en el Pacífico oriental durante los últimos 65 m.a. tienden a ser dominadas por especies pertenecientes a un reducido número de grupos funcionales y pudieron tener altos niveles de redundancia ecológica (Clarke y Warwick, 1998). Es interesante denotar que aun cuando en el Reciente se tienen 42 especies en la región, se ha hecho patente la vulnerabilidad de los corales a fenómenos como el calentamiento del mar, derivado de la Oscilación Sureña de El Niño (Glynn, 2001), en parte resultante de la semejanza en el tipo de zooxantelas presente en las especies del mismo género (Baker, 2003), es decir, de una amplitud limitada en el nicho ecológico.

En conclusión, el índice de discrepancia taxonómica $\left(\Delta^{+}\right)$de los corales zooxantelados del Pacífico orien- 
tal mostró notable estabilidad a lo largo del Cenozoico y no pareció verse afectado por los eventos masivos de extinción y originación, como ocurrió con la riqueza de especies. Por otra parte, la riqueza y la variación de la discrepancia taxonómica $(\Lambda+)$ se comportaron de manera inversa debido a que en épocas geológicas cuando hubo alta riqueza se caracterizan por comunidades con especies taxonómicamente afines, que podría ser indicativo de una redundancia ecológica alta en los ecosistemas arrecifales de la región estudiada y una resistencia potencialmente menor a cambios ambientales en las épocas de mayor variedad específica.

\section{Agradecimientos}

Este trabajo se desarrolló con el apoyo de SEP-PROMEP (proyecto UABCS 003-2004, asignado al primer autor) y con fondos de la Universidad de Iowa, Sociedad Geológica de América, el Fondo Max y Lorraine Littlefield y mediante una beca de estudios de doctorado (CONACYT 110226) asignados al segundo autor. Se agradecen los comentarios para mejorar el contenido del estudio por parte de Gerardo Barba (UABCS, Museo de Historia Natural) y de los revisores anónimos.

\section{Literatura citada}

Baker, A. C. 2003. Flexibility and specificity in coral -algal symbiosis: diversity, ecology and biogeography of Symbiodinium. Annual Review of Ecology and Systematics 34:661-689.

Baron-Szabo, R. C. 2008. Corals of the K/T-boundary: scleractinian corals of the suborders Dendrophylliina, Caryophylliina, Fungiina, Microsolenina, and Stylinina. Zootaxa 1952:1-244.

Budd, A. F. 1991. Neogene paleontology in the northern Dominican Republic, 11. The Family Faviidae (Anthozoa: Scleractinia). Part I. The Genera Montastraea and Solenastrea. Bulletins of American Paleontology 101:5-83.

Budd, A. F. 2000. Diversity and extinction in the Cenozoic history of Caribbean reefs. Coral Reefs 19:25-35.

Budd, A. F. y K. G. Johnson 1999. Neogene paleontology in the northern Dominican Republic. 19. The Family Faviidae (Anthozoa: Scleractinia), pt. II. The genera Caulastrea, Favia, Diploria, Thysanus, Hadrophyllia, Manicina and Colpophyllia. Bulletins of American Paleontology 356:1-83.

Budd, A. F. y J. M. Pandolfi. 2010. Evolutionary novelty is concentrated at the edge of coral species distributions. Science 328:1558-1561.

Clarke, K. R. y R. M. Warwick. 1998. Quantifying structural redundancy in ecological communities. Oecologia 113;278289.
Clarke, K. R. y R. M. Warwick. 2001. A further biodiversity index applicable to species lists: variation in taxonomic distinctness. Marine Ecology Progress Series 216:265-278.

Campbell, B. W. y R. Novelo-Gutiérrez. 2007. Reduction in odonate phylogenetic diversity associated with dam impoundment is revealed using taxonomic distinctness. Fundamental and Applied Limnology 168:83-92.

Colgan, M. W. 1990. El Niño and the history of Eastern Pacific reef building. In Global ecological consequences of the 1982-83 El Niño Southern Oscillation, P. W. Glynn (ed.). Elsevier, Amsterdam. p. 183-232.

Durham, J. W. 1947. Corals from the Gulf of California and the North Pacific Coast of America. Geological Society of America Memoir 20:1-68.

Euler, F. V. y S. Svensson. 2001. Taxonomic distinctness and species richness as measures of functional structure in bird assemblages. Oecologia 129:304-311.

Foster, A. B. 1986. Neogene paleontology in the Northern Dominican Republic. 3. The family Poritidae (Anthozoa: Scleractinia). Bulletins of American Paleontology 90:45-123.

Foster, A. B. 1987. Neogene Paleontology in the Northern Dominican Republic. 4. The genus Stephanocoenia (Anthozoa: Scleractinia: Astrocoeniidae). Bulletins of American Paleontology 93:5-22.

Glynn, P. W. 2001. Eastern Pacific coral reef ecosystems. In Coastal marine ecosystems of Latin America, U. Seeliger y B. Kjerfve (eds.). Springer, Berlin. p. 281-305.

Glynn, P. W. y J. S. Ault. 2000. A biogeographic analysis and review of the far Eastern Pacific coral reef region. Coral Reefs 19:1-23.

Graham, N. A. J., S. K. Wilson, S. Jennings, N. V. C. Polunin, J. P. Bijoux y J. Robinson. 2006. Dynamic fragility of oceanic coral reef ecosystems. Proceedings of the National Academy of Sciences 103:8425-8429.

Grigg, R. W. y R. Hey. 1992. Paleoceanography of the tropical eastern Pacific. Science 255:172-178.

Johnson, K. G. 2001. Middle Miocene recovery of Caribbean reef corals: new data from the Tamana Formation, Trinidad. Journal of Paleontology 75:513-526.

Johnson, K. G., J. B. C. Jackson y A. F. Budd. 2008. Caribbean reef development was independent of coral diversity over 28 million years. Science 319:1521-1523.

Johnson, M. E., R. A. López-Pérez, C. R. Ransom y J. LedesmaVázquez. 2007. Late Pleistocene coral-reef development on Isla Coronados, Gulf of California. Ciencias Marinas 33:1-16.

Kiessling, W. y R. C. Baron-Szabo. 2004. Extinction and recovery patterns of scleractinian corals at the CretaceousTertiary boundary. Palaeogeography, Palaeoclimatology, Palaeoecology 204:195-223.

López-Pérez, R. A. 2005. The Cenozoic hermatypic corals in the eastern Pacific: history of research. Earth-Science Reviews 72:67-87. 
López-Pérez, R. A. 2008. Fossil corals from the Gulf of California, Mexico: still a depauperate fauna but it bears more species than previously thought. Proceedings of the California Academy of Science series 4 59:515-531.

López-Pérez, R. A. 2012. Late Miocene to Pleistocene reef corals in the Gulf of California. Bulletins of American Paleontology $382: 1-78$.

López-Pérez, R. A. y A. F Budd. 2009. Coral diversification in the Gulf of California during Late Miocene to Pleistocene. In Atlas of coastal ecosystems in the Gulf of California: past and present, M. E. Johnson y J. Ledesma-Vasquez (eds.). University of Arizona Press, Tucson. p. 58-71.

López-Pérez, R. A. y H. Reyes-Bonilla. 2000. Los corales constructores de arrecifes en el Pacífico mexicano. Una historia de 65 millones de años. Ciencia 51:4-12.

Magurran, A. E. 2004. Measuring biological diversity. Blackwell, Cambridge, Massachusetts. 256 p.

Pandolfi, J. M. 2011. The paleoecology of coral reefs. In Coral reefs: an ecosystem in transition, Z. Dubinski y N. Stambler (eds.). Springer, Berlin. p. 13-24.

Reyes-Bonilla, H. 2002. Checklist of valid names and synonyms of stony corals (Anthozoa: Scleractinia) from the Eastern Pacific. Journal of Natural History 36:1-13.

Reyes-Bonilla, H., L. E. Calderón-Aguilera, G. Cruz-Piñón, P. Medina-Rosas, R. A. López-Pérez, M. D. Herrero-Pérezrul, G. E. Leyte-Morales, A. L. Cupul-Magaña y J. D. CarriquiryBeltrán. 2005. Atlas de los corales pétreos (Anthozoa: Scleractinia) del Pacífico mexicano. Centro de Investigación
Científica y de Educación Superior de Ensenada, CONABIO/ CONACYT/ Universidad de Guadalajara/ Centro Universitario de la Costa, Universidad del Mar, Guadalajara, Jalisco. 128 p.

Rogers, S. I., K. R. Clarke y J. D. Reynolds. 1999. The taxonomic distinctness of coastal bottom-dwelling fish communities of the north-east Atlantic. Journal of Animal Ecology 68:769782 .

Schweiger, O., S. Klotz, W. Durka y I. Kuhn. 2008. A comparative test of phylogenetic diversity indices. Oecologia 157:485495.

Vaughan, T. W. 1917. The coral reef fauna of Carrizo Creek, Imperial County, California, and its significance. United States Geological Survey Professional Paper 98:355-386.

Veron, J. E. N., 1995. Corals in space and time. Comstock, Cornell, Ithaca. $332 \mathrm{p}$.

Warwick, R. M. y K. R. Clarke. 1995. New 'biodiversity' measures reveal a decrease in taxonomic distinctness with increasing stress. Marine Ecology Progress Series 129:301305.

Warwick, R. M. y K. R. Clarke. 1998. A taxonomic distinctness index and its statistical properties. Journal of Applied Ecology 35:523-531.

Warwick, R. M. y K. R. Clarke. 2001. Practical measures of marine biodiversity based on relatedness of species. Oceanography and Marine Biology Annual Review 39:207-231.

Zar, J. H. 2009. Biostatistical analysis, quinta edición. Prentice Hall, Englaewood Cliffs, New Jersey. 960 p. 